\title{
High Performance Wireless Sensor Network Localization System
}

\section{Michał Marks}

Research and Academic Computer Network (NASK)

Wawozowa 18, 02-796 Warsaw, Poland

E-mail: mmarks@elka.pw.edu.pl

\section{Ewa Niewiadomska-Szynkiewicz}

Institute of Control and Computation Engineering, Warsaw University of Technology

Nowowiejska 15/19, 00-665 Warsaw, Poland

Research and Academic Computer Network (NASK)

Wawozowa 18, 02-796 Warsaw, Poland

E-mail: ens@ia.pw.edu.pl

\section{Joanna Kołodziej}

Institute of Computer Science, Cracow University of Technology Warszawska 24, 31-155 Cracow, Poland

E-mail: jokolodziej@pk.edu.pl

\begin{abstract}
:
In this paper we summarize the results of our research concerned with the development, implementation and evaluation of a software framework for wireless sensor networks localization - High Performance Localization System (HPLS). The system can be used to calculate positions of sensing devices (network nodes) in the deployment area, and to tune and verify various localization schemes through simulation. It provides tools for data acquisition from a workspace, estimation of inter-node distances, calculation of geographical coordinates of all nodes with unknown position and results evaluation. Received Signal Strength measurements are utilized to support the localization process. Trilateration, simulated annealing and genetic algorithm are applied to calculate the geographical coordinates of network nodes. The utility, efficiency and scalability of the proposed localization system HPLS have been justified through simulation and testbed implementation. The calculations have been done in parallel using the map-reduce paradigm and the HPC environment formed by a cluster of servers. The testbed networks were formed by sensor devices manufactured by Advantic Technology (clones of TelosB platform). A provided case study demonstrates the localization accuracy obtained for small-, medium- and large-size multihop networks.
\end{abstract}

Keywords: Wireless Sensor Networks, localization, positioning, stochastic optimization, real life deployments, HPC, testbed verification.

\section{Introduction}

Advances in wireless communication and electronics have enabled the design and development of tiny, multifunctional and energy-aware devices providing sensing, computing and communicating capabilities. These devices can be standalone or can be integrated and deployed to form a Wireless Sensor Network (WSN) in support to a variety of civil, environmental and military applications. Recently, it is observed that monitoring and control of physical environments become a hot spot in the technology landscape. New sensing devices with different phenomena's monitoring capabilities are offered by a number of companies. They are capable to communicate with each other and in a multihop way propagate sensing information to a base station that can play a role of the operation center. While the set of challenges in sensor networks are diverse, researches have mainly focused on fundamental challenges, which include: planning, optimal sensor deployment, localization, reliable and energy-aware inter-node communication. In the last few years, wireless sensor networking has been a very active research field in both academia and the industry with a wide variety of 
applications and research projects. An extensive survey of the state of the art in WSN programming approaches and WSN applications is provided in literature Akyildiz \& Vuran (2010), Beutel (2005), Farooqi \& Khan (2012), Karl \& Willig (2005), Rodrigues \& Neves (2010), Wang et al. (2010).

However, the performance of a WSN strongly depends on the way sensor devices were positioned in the area. Moreover, many applications of wireless sensor networks, e.g., monitoring, target tracking, search, etc., require the correlation of sensor measurements with physical locations. Even if the accessible knowledge about positions of nodes is only approximate, there are great opportunities for use of various network services, location-based routing, data aggregation, etc. In general, self-organization and localization capabilities are one of the most important requirements in sensor networks.

The goal of localization is to assign geographical coordinates to each node with unknown position in the deployment area. The location of sensor devices can be obtained in two ways: 1) recording data on the geographical coordinates of sensors during their deployment in a workspace, 2) fitting sensors with a GPS system. Both methods have significant drawbacks. Manually distributing and recording positions of each sensor device is impractical for large networks. In many applications, sensors are distributed randomly in ad hoc fashion, which is cheaper, and in some cases the only possible solution. Another approach is to use the GPS system to collect data on the location of sensors. Although this solution can be used in different types of networks, it is very costly, both due to the price of GPS, and to the increased power consumption that may decrease the lifetime of a WSN. Moreover, adding an extra device increases the size and weight of the network node. Due to the drawbacks of both mentioned solutions, numerous automated systems to localization in WSN have been developed. With regard to limited resources of a WSN all localization schemes have to work with minimal energy requirements, scale to large networks, and also achieve good accuracy in the presence of interference and irregularities and give the solution in the reasonable time.

The localization techniques can be classified with respect to various criterion (see NiewiadomskaSzynkiewicz (2012)). They differ on hardware's capabilities, nodes properties and deployment, measurement and calculation methods, computing organization and assumed precision, etc. Many localization strategies estimate positions of nodes based on merely partial knowledge of the location of the set of nodes (anchors) in a network and measurements of inter-node distances. An anchor (also known as a beacon node) is defined as a node that is aware of its own location, either through manual preprogramming during deployment or the GPS system. With regard to hardware's capabilities of sensor devices inter-node distances can be estimated based only on connectivity information (range free localization) or
Euclidean distance calculated based on radio signal strength measurements or angle estimates (range based localization). The common range free methods are hop-counting techniques. The range based methods often require additional equipment such as antenna, signal generator, etc. In accordance with the available hardware they exploit angle of arrival (AoA), time of arrival (ToA), time difference of arrival of two different signals (TDoA) and received signal strength indicator (RSSI). The survey of these technologies is provided in Akyildiz \& Vuran (2010), Barsocchi et al. (2009), Benkic et al. (2008), Karl \& Willig (2005), Motter et al. (2011).

We focus on the range based techniques and application of global optimization solvers to estimate geographical coordinates assigned to network nodes. In our approach the localization problem is formulated as a nonlinear optimization problem. In this paper we compare five localization systems utilizing algorithms: Trilateration (T), Simulated Annealing (SA), Genetic Algorithm (GA), and two hybrid schemes that use a combination of a geometry of triangles, along with a stochastic optimization - Trilateration \& Simulated Annealing (TSA) and Trilateration \& Genetic Algorithm (TGA).

The main contribution of the paper is to present a software framework - High Performance Localization System (HPLS) - that provides tools to localization of wireless sensor devices that form various types of networks. The system implements mentioned above localization methods. It can operate in two modes. In the on-line mode the system is used to calculate a location of each device with unknown position in the real life sensor network. In the off-line mode it can be used to verify, evaluate and compare the performance of various localization schemes that are integrated with the framework.

In general, WSN localization is a complex task and usually involves cumbersome calculations, especially when consider large-size networks with hundreds or thousands of nodes. Moreover, it was observed (see Niewiadomska-Szynkiewicz \& Marks (2009), Niewiadomska-Szynkiewicz (2012)) that different methods are suitable for different network topologies. Therefore, development of high accuracy localization system that can be applied to various networks requires multiple execution of the localization schemes for different values of parameters specific to the considered algorithms and various test networks. Therefore, High Performance Computing (HPC) techniques are employed in the HPLS to speed up localization of WSNs.

The HPLS has been validated for a numerous synthetic and real life wireless sensor networks. The results of experiments show both the utility and efficiency of the framework and accuracy and scalability of the provided localization algorithms.

The paper is structured as follows. Section 2 gives a short overview of localization methods and systems. The localization problem formulation and description 
of techniques used to calculate inter-node distances are presented in Section 3. In Section 4 an overview of the HPLS is provided. The results of the HPLS performance evaluation and the comparison of selected localization schemes through simulation and testbed implementation are described in Sections 5 and 6. Finally, conclusions are drawn in Section 7.

\section{Related Works}

Numerous systems for WSNs localization have been developed. An overview of various approaches and attempts to classify different techniques is presented in the literature: Akyildiz \& Vuran (2010), Ji (2011), Niewiadomska-Szynkiewicz (2012), Anderson et al. (2007), Mao \& Fidan (2009), Sarigiannidis (2007). The localization strategies can generally be divided into coarse-grained localization techniques that consist of finding approximate coordinates of nodes in a network and fine-grained strategies that determine the exact positions of unknown nodes for exact distance measurements but require much more communication and computation. Various techniques are used to estimate the geographical coordinates of sensor devices. The commonly used simple methods are geometrical techniques, i.e., triangulation, trilateration and multitrilateration reported in Akyildiz \& Vuran (2010), Beutel (2005). A number of protocols and location systems implementing these techniques have been developed. The simple system implementing the trilateration method is called Ad-hoc Positioning System (APS), and is described in Niculescu \& Nath (2001). Two versions of APS - range free (DV-hop) and range based (DV-distance) are proposed. Another systems - Ad Hoc Localization System (AHLoS) described in Savvides et al. (2001) and the system for dynamic indoor localization described in Wessels et al. (2010) apply the multitrilateration method to calculate the position of nodes in a network.

Other popular range free and range based location systems utilize Multidimensional Scaling (MDS) to the localization of nodes. In general, MDS is a set of data analysis techniques used for exploring similarities or dissimilarities in data Mao \& Fidan (2009). The philosophy of localization techniques based on MDS is to formulate a mathematical model to convert distance information into the coordinate vector. A number of MDS-based localization systems have been developed and described in the literature Costa et al. (2006), Shang et al. (2004), Vo et al. (2008). Some of them utilize clustering techniques Medidi et al. (2006), Shu et al. (2009).

Recently, popular approach to WSN localization is to formulate an optimization problem, and solve it using linear or nonlinear solvers. The standard formulation is a nonconvex optimization problem with nonlinear performance function. Various optimization techniques are used to solve such optimization problem. The most popular approaches are: quadratic, linear and nonlinear programming. The first class of methods relax the original nonconvex problem in order to obtain a quadratic one. The range free localization system OPDMQP using quadratic programming is described in Lee et al. (2010). The widely used technique is to transform the nonconvex problem into a standard semidefinite programming (SDP) problem and use the linear solvers to solve the relaxed problem. The formulation of SDP localization problem is provided in Biswas \& Ye (2004). The SDP-based localization systems are described in Tseng (2007), Wang et al. (2008). The application of lagrangian multiplied method to the localization problem is described in $\mathrm{Li}$ et al. (2010). The common strategy is to apply heuristics to solve the localization problem. The localization systems employing deterministic and stochastic heuristics such as: tabu search Shekofteh et al. (2010), simulated annealing Niewiadomska-Szynkiewicz \& Marks (2009), Kannan et al. (2005, 2006), genetic algorithm Niewiadomska-Szynkiewicz \& Marks (2009), evolutionary algorithm Sayadnavard et al. (2010), Vecchio et al. (2012) and particle swarm optimization Chuang \& Wu (2008) have been developed.

Another solutions are hybrid schemes that combine various methods for computing the coordinate vector. In most approaches trilateration or multitrilateration is used to calculate an initial solution, which is improved in the second step, see Tam et al. (2006), NiewiadomskaSzynkiewicz \& Marks (2009). Optimization algorithms are usually employed in this step to calculate the final solution. Hybrid technique seems to be a good solutions to speed up the calculation process without decreasing the accuracy of the location estimation.

\section{Range Based Localization}

We have developed two hybrid schemes - two-phase range based methods that combine trilateration, along with a stochastic optimization for performing sensor localization. The received signal strength measurements are utilized to inter-node distances estimation. In this section we present the formulation of our model and describe calculation methods. We start from the description of the network to be localized, and then we present techniques applied to inter-node distances estimation and geographical coordinates calculation provided in the HPLS.

\subsection{Network System Definition}

Let us assume that the network is a physical system modeled as a set $S_{s}$ of wireless sensor devices (network nodes) $s_{i}, i=1, \ldots, I$, each with position expressed as $l$-dimensional coordinates $s_{i} \in \mathbb{R}^{l}$. The set $S_{s}$ consists of two types of devices: $M$ anchor nodes, each with known position in a workspace expressed as $l$-dimensional coordinates $a_{k} \in \mathbb{R}^{l}, k=1, \ldots, M$, and $N$ non-anchor 
nodes $x_{j} \in \mathbb{R}^{l}, j=1, \ldots, N$ with unknown locations. We can introduce an Euclidean inter-node distances for all pairs of nodes in the network, i.e., $d_{k j}=\| a_{k}-$ $x_{j} \|$ between anchors and non-anchors, and $d_{i j}=\| x_{i}-$ $x_{j} \|$ between two non-anchors, and $i \neq j$. In most practical applications of range based methods, each sensor emits a known signal that allows neighboring nodes to estimate their distances. Therefore, we can define sets of neighboring nodes as collections of nodes located within radio ranges of given nodes. Hence, we introduce the following sets for all anchor and nonanchor nodes:

$$
\begin{gathered}
N_{k}=\left\{(k, j): d_{k j} \leq r_{k}\right\}, \quad j=1, \ldots, I, \\
N_{i}=\left\{(i, j): d_{i j} \leq r_{i}\right\}, \quad j=1, \ldots, I,
\end{gathered}
$$

where $r_{k}$ and $r_{i}$ denote the maximal transmission ranges of $k$-th anchor node and $i$-th non-anchor node.

\subsection{Inter-node Distances Calculation}

The range based localization schemes use absolute internode distance estimates or angle estimates in location calculation. In real life applications the estimates $\tilde{d}_{k j}$ and $\tilde{d}_{i j}$ of true physical distances $d_{k j}$ and $d_{i j}$ are produced by various measurement methods (see Akyildiz \& Vuran (2010), Barsocchi et al. (2009), Benkic et al. (2008), Beutel (2005), Karl \& Willig (2005), Motter et al. (2011)). In case of devices equipped with the radio transceiver the RSSI (Received Signal Strength Indicator) can be successfully used to estimate distances. Our localization system (HPLS) implements the RSSI method due to low cost (no additional hardware), easy configuration and deployment. The disadvantage of this solution is low level of measurement accuracy because of high variability of the RSSI value Benkic et al. (2008), Ramadurai \& Sichitiu (2003). Nevertheless, many authors indicate that new radio transceivers can give RSSI measurements good enough to be a reasonable distance estimator Barsocchi et al. (2009), Srinivasan \& Levis (2006).

We have used the radio signal propagation model outlined in Marks \& Niewiadomska-Szynkiewicz (2011), Rappapport (2002) to transform RSSI measurements into inter-node distances. This model indicates that received signal power decreases with a distance, both in outdoor and indoor workspaces. Therefore, the power of the signal received by a receiver $P^{r}$ at a distance $d$ between a sender and a receiver can be defined as follows:

$$
P^{r}(d)[d B m]=P^{t}[d B m]-P L(d)[d B],
$$

where $P^{t}$ denotes power used by a sender to transmit the signal and $P L(d)$ (path loss) the average degradation of a signal with a distance $d . P L(d)$ in $(3)$ is modeled as a function of a distance $d$ raised to an attenuation constant $n$ that indicates the rate, at which the path loss increases with a distance:

$$
P L(d)[d B]=P L\left(d_{0}\right)[d B]+10 n \log \left(\frac{d}{d_{0}}\right),
$$

where $d_{0}$ is a close-in reference distance (for IEEE 802.15.4 usually $d_{0}=1 \mathrm{~m}$ ). The formulas (3) and (4) can be used to estimate the average distance $\tilde{d}_{i j}$ between each pair of nodes $(i, j)$ in a network. Hence, the estimate of each inter-node distance is a function of the received signal strength:

$$
\tilde{d}_{i j}=d_{0} \cdot 10^{\frac{P^{t}-P L\left(d_{0}\right)}{10 n}} \cdot 10^{-\frac{P_{i j}^{r}}{10 n}}=\eta \cdot 10^{\theta \cdot P_{i j}^{r}},
$$

where $P L\left(d_{0}\right)$ denotes the path loss at the reference distance $d_{0}, P^{t}$ output power of the transmitter, $P_{i j}^{r}$ received signal strength measured for each pair $(i, j)$ of nodes, $\eta=d_{0} \cdot 10^{\frac{P^{t}-P L\left(d_{0}\right)}{10 n}}$ and $\theta=-\frac{1}{10 n}$.

In summary, in on-line localization of real life networks we can estimate the distances between each pair of non-anchor nodes and distances between each pair consisting of anchor and non-anchor node using RSSI.

\subsection{Geographical Coordinates Calculation}

The next step of our localization process is to calculate the geographical coordinates. Hence, the general localization problem statement is as follows. Given noisy distance measurements $\tilde{d}_{k j}, \tilde{d}_{i j}$ and positions of anchor nodes $a_{k} \in \mathbb{R}^{l}, k=1, \ldots, M$ estimate the locations of all nodes $x_{j} \in \mathbb{R}^{l}, j=1, \ldots, N$ with unknown positions. We can formulate the model of the optimization problem that minimizes the sum of errors in sensor positions for fitting the distance measurements. This model can be described as follows:

$$
\begin{aligned}
\min _{\hat{x}}\left\{J=\sum_{k=1}^{M} \sum_{j \in N_{k}}\left(\left\|a_{k}-\hat{x}_{j}\right\|_{2}-\tilde{d}_{k j}\right)^{2}\right. & \\
& \left.+\sum_{i=1}^{N} \sum_{j \in N_{i}}\left(\left\|\hat{x}_{i}-\hat{x}_{j}\right\|_{2}-\tilde{d}_{i j}\right)^{2}\right\},
\end{aligned}
$$

where $\hat{x}_{i}$ and $\hat{x}_{j}$ denote estimated positions of nodes $i$ and $j, \tilde{d}_{k j}$ and $\tilde{d}_{i j}$ distances between pairs of nodes $(k, j)$ and $(i, j)$ calculated according to the method described in Section 3.2, $N_{k}$ and $N_{i}$ sets of neighbors of anchor and non-anchor nodes defined in (2).

We propose to use SA or simple GA to solve the problem (6). In case of hybrid schemes the optimization algorithm is executed after preliminary trilateration.

\section{Localization System Overview}

The High Performance Localization System (HPLS) is a parallel software framework to localization of wireless sensor devices that form various types of networks. Moreover, it is a powerful and scalable platform for testing various localization schemes on parallel computers or computer clusters. The current version of the system implements several localization schemes. Due to an open architecture the HPLS can be easily extended with implementations of new approaches to localization of nodes in a network. 
Figure 1 The components of the HPLS.

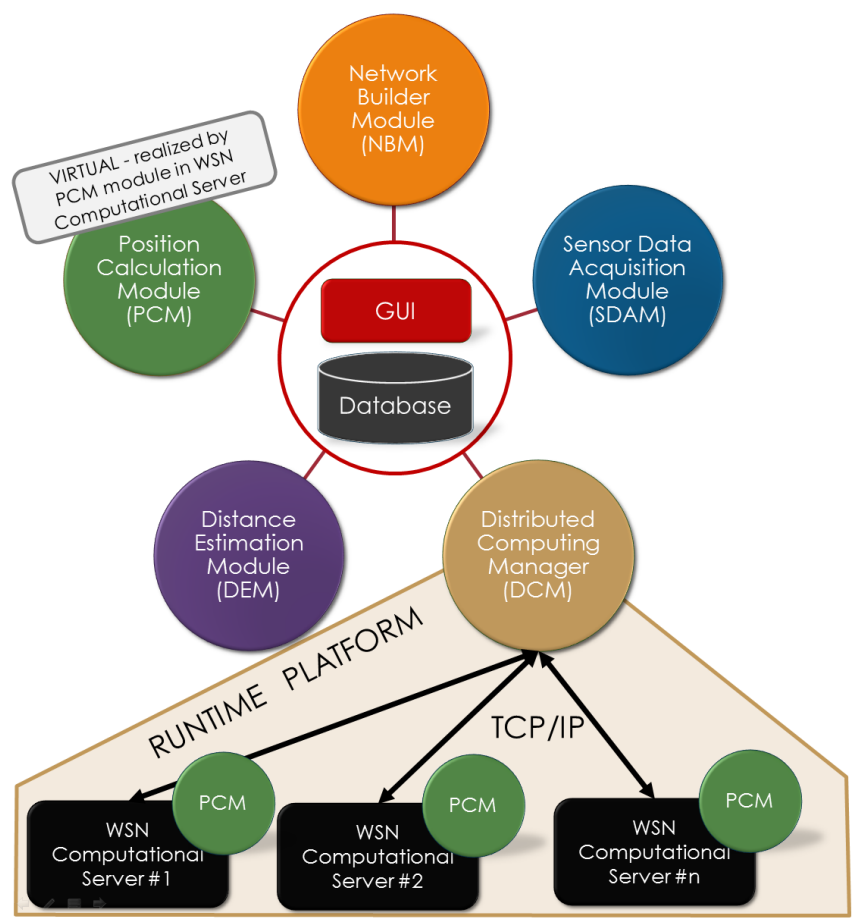

\subsection{HPLS Architecture}

The HPLS is composed of five main components (see Fig. 1):

- RP: Runtime Platform.

- SDAM: Sensor Data Acquisition Module.

- NBM: Network Builder Module.

- DEM: Distance Estimation Module.

- PCM: Position Calculation Module.

The enumerated modules are described in the following subsections. Moreover, two additional components are provided: Database (DB) for recording data of all examined networks and results of calculations and GUI that provides tools for supporting the interaction with a user.

\subsection{Runtime Platform}

The Runtime Platform (RP) is the system kernel that manages calculations and communication between running processes. RP provides an environment for effective computing on a parallel computer or a cluster of workstations. It is formed by two components: Distributed Computing Manager (DCM) and Computational Server (CS). The DCM module is responsible for splitting each calculation task into subtasks and allocating these subtasks into processors or computers. Moreover, DCM is responsible for managing the calculations, interprocess communication and load
Figure 2 Architecture of the Computational Server (CS).

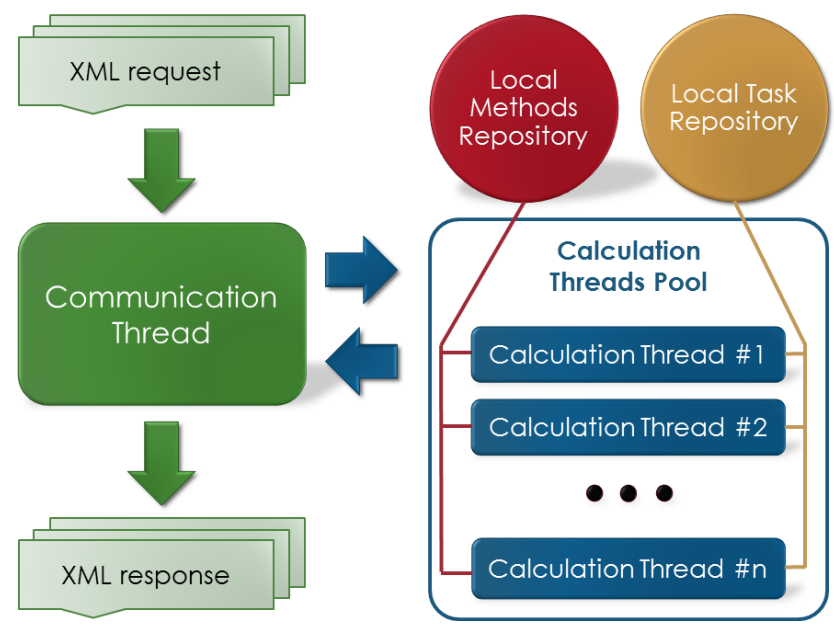

balancing. Each DCM module can be used to manage many computational servers.

The Computational Server (CS) is responsible for managing the calculation of location of sensor devices in a workspace. Sequentially, the following operations are performed: all initial parameters specified to solvers, and given WSN topology are loaded, next the module responsible for location calculation is executed and the results of calculations - estimated positions of nodes are sent to GUI. To speedup the preliminary tuning of a localization solver to a given WSN application, and testing various localization schemes the multithread computing is performed. Many CS modules can be executed simultaneously, each on different, remote machine. The number of threads should not exceed the number of processor cores in the computing system. The XML configuration file stores the information about a number of available cores, a communication port and IP address. Each CS module provides two local repositories that contain a collection of localization methods and the networks to be localized. The architecture of the Computational Server is presented in Fig. 2.

The client-server communication model is used for inter-node communication. The communication between the DCS and each CS is based on TCP/IP and BSD sockets. The communication protocol is implemented in the XML scheme. The idea of this protocol was to develop the simple mechanism that fulfills flexibility and failure resistance.

\subsection{Sensor Data Acquisition Module}

The Sensor Data Acquisition Module (SDAM) is responsible for data gathering from real life deployments of sensor devices in a workspace. The following measurements are necessary to determine positions of nodes utilizing methods described in Section $3.2-$ geographical coordinates of anchor nodes and RSSI measurements for all pairs of nodes. The SDAM operates in two steps. In the first one - manual, the user 
Figure 3 Scheme of the data acquisition system.

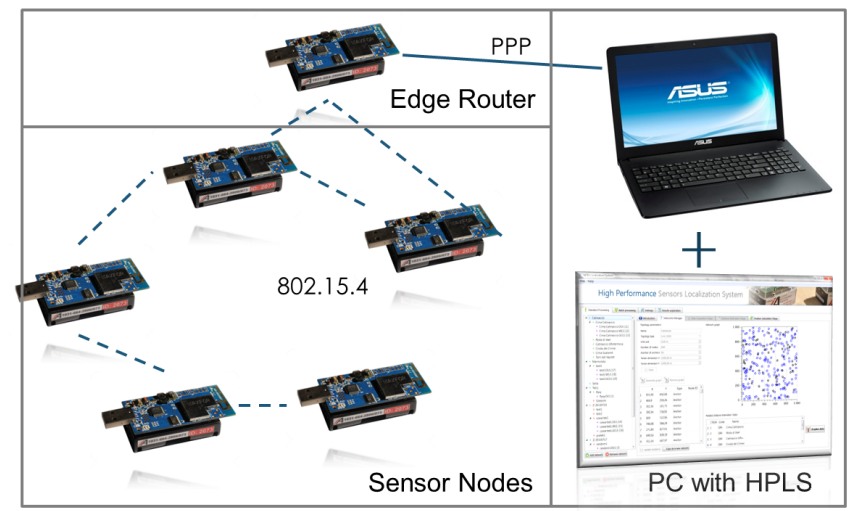

has to provide some necessary data, i.e., number of anchor nodes, coordinate vectors of anchor nodes (only if the data are gathered from a network with anchor nodes deployed manually). In the second step all RSSI measurements are acquired automatically. The SDAM collects data from the network through a selected sensor, which plays a role of an edge router. The edge router is connected to the HPLS via PPP protocol (over USB connector) and has the capability to communicate with other sensor nodes using the IEEE 802.15.4 protocol. The data gathering process is depicted in Fig. 3 .

The data collection protocol is based on BLIP 2.0 stack. BLIP 2.0 - Berkeley Low-power IP stack, is the reimplementation in TinyOS of a number of IP-based protocols including IPv6, RPL and CoAP. Detailed description can be found in Silva et al. (2009), Rodrigues $\&$ Neves (2010). It is assumed that each sensor node in a network is responsible for exchanging packets with all nodes in its neighbourhood and collecting a vector of pairs neighbour $i d$ and RSSI measurement. Next, these vectors are sent using multi-hop unicast transmission to the edge router and then registered in the HPLS database.

\subsection{Network Builder Module}

The aim of the Network Builder Module (NBM) is to create and store a network to be localized. Two scenarios are available due to possible applications of the HPLS.

In the first scenario, the real life wireless sensor network may is mapped in the HPLS database. All data about sensors deployments and measurements are provided by the SDAM.

The second scenario is to create synthetic networks for the purpose of simulations. The NBM implements a network generator that is responsible for modeling networks with various low-power topologies. A user can add, remove and modify networks by selecting appropriate topology, channel and radio parameters. Low-power link modeling provided by HPLS is based on Link Layer Model for MATLAB described in Zuniga \& Krishnamachari (2004). The user can define parameters for generated networks concerning both channel and
Figure 4 Part of the HPLS GUI responsible for setting simulation parameters.

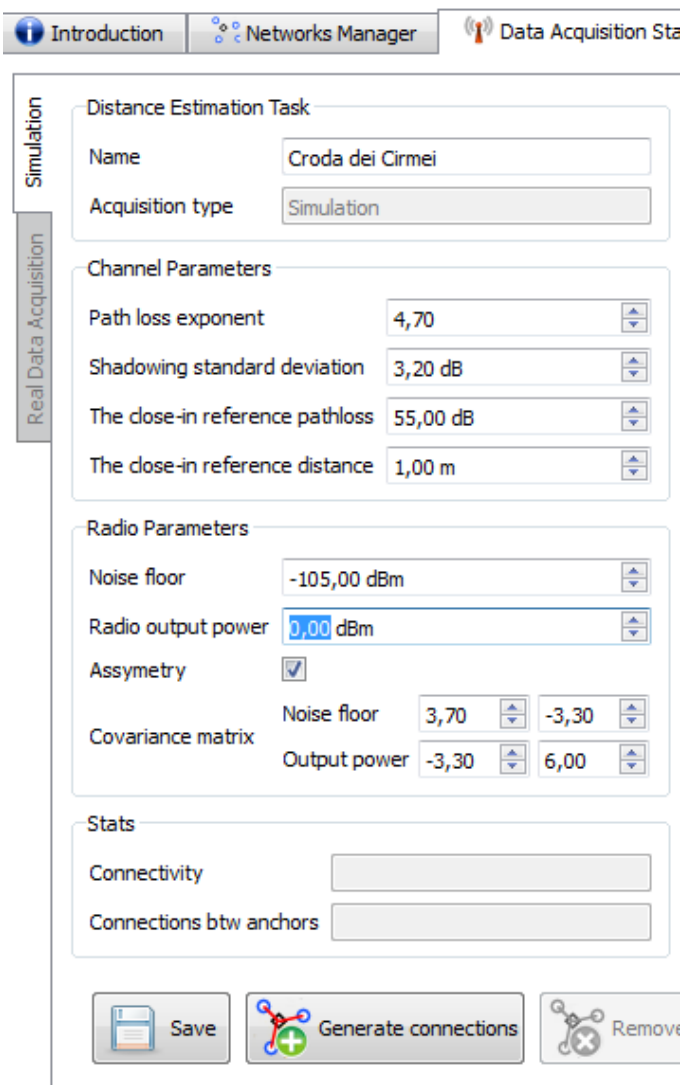

radio modelling, as it can be find in Fig. 4. Channel modelling is mostly dependant on the environment where the WSN network is deployed. In our real-life deployments (outdoor, on the ground/grass) we observed typical pathloss for reference distance between 45 and $55 \mathrm{~dB}$ depending on altitude of sensor mounting and such parameters can be applied for simulating network with bigger number of nodes. The simulation module allows on quite accurate radio behaviour modelling for example we can generate networks with RSS receptions characteristic for CC2420 radio chip used in our testbed like power transmission equal $-25 \mathrm{dBm}$ and noise level equal $-95 \mathrm{dBm}$.

\subsection{Distance Estimation Module}

The Distance Estimation Module (DEM) transforms RSSI measurements gathered by the SDAM into inter-node distance estimates using the radio signal propagation model presented in Section 3.2. Three approaches to distance estimation are provided: Ordinary Least Square Method (OLS), Weighted Least Square Method (WLS) and Geometric Combined Least Square Method (GCLS). Ale three methods utilize stochastic optimization methods solving global optimization problem with different formulations of performance measures. They are described in details and evaluated in Marks \& Niewiadomska-Szynkiewicz (2011). 


\subsection{Position Calculation Module}

The aim of the Position Calculation Module (PCM) is to calculate the coordinates of non-anchor nodes in the network using inter-node distances. The PCM provides a library of methods that can be used to solve the localization problem (6). The current version of this library consists of five techniques: simple Trilateration (T), Simulated Annealing (SA), Genetic Algorithm (GA), Trilateration \& Simulated Annealing (TSA) and Trilateration \& Genetic Algorithm (TGA).

The fastest and the most popular localization technique is Trilateration. There are many attempts in the literature how to use geometry of triangles to locate the motes in the network, e.g. Niculescu \& Nath (2001). However it is obvious that the quality of such solution is not especially good because of the noise in the measurements. That is why more sophisticated methods are provided like: SA, GA or hybrid techniques (TSA, TGA). Although the evolutionary or genetic algorithms are recognized as faster approaches we decided to provide also simulated annealing as the structure of the localization task is similar to travelling salesman problem (TSP).

The general scheme of the SA algorithm described in Mao et al. (2007) with some modifications was implemented and is provided in the PCM library. In our implementation the cooling process is slowed down w.r.t. the basic SA algorithm. At each value of the coordinating parameter $T$ (temperature), not one but $P \cdot N$ nonanchor nodes are randomly selected for modification (where $P$ is a reasonably large number to make the system into thermal equilibrium). The efficiency and robustness of this algorithm strongly depend on control parameters of the algorithm. All these parameters influence the speed of convergence and accuracy of the solution. To obtain the general purpose algorithm all parameters have to be tuned up for diverse network topologies.

The scheme of the GA algorithm presented in Goldberg (1989) was implemented and is provided in the PCM library. The abstract representations of candidate solutions (chromosomes) are vectors of coordinates of all non-anchor nodes: $\left[x_{1}^{1}, \ldots, x_{1}^{l}, x_{2}^{1}, \ldots, x_{2}^{l}, \ldots\right.$, $\left.x_{N}^{1}, \ldots, x_{N}^{l}\right], x_{i}^{j} \in \mathbb{R}$ where $i=1, \ldots, N, j=1, \ldots, l$. The initial population consists of a set of such chromosomes. The fitness function is defined in (6). The tournament selection of size equal two is used at the reproduction stage. The crossover operator is defined as discrete recombination similar to elements exchanging applied to binary vectors. Both coordinates are recombined simultaneously. The mutation operator modifies the components of a given chromosome by adding a vector of generated $l \cdot N$ Gaussian random variables. The elitist succession model is chosen. The GA algorithm efficiency and robustness strongly depend on a size of population and control parameters specific to the genetic operators.
The PCM provides implementations of two hybrid methods: TSA that combines multitrilateration and simulated annealing and TGA that combines multitrilateration and genetic algorithm. These localization methods operate in two phases: 1) the auxiliary solution (localization) is provided using the geometry of triangles, 2) the results of initial localization are refined using the stochastic optimization (simulated annealing in TSA and genetic algorithm in TGA). The additional functionality (correction) is introduced to the second phase of the algorithm to remove incorrect solutions involved by the distances measurement errors. The detailed description of TSA and TGA methods is provided in Niewiadomska-Szynkiewicz \& Marks (2009). The screenshot of Graphical User Interface illustrated operation of Runtime Platform with distributed PCM modules is presented in Figure 5.

Figure 5 The HPLS graphical user interface.

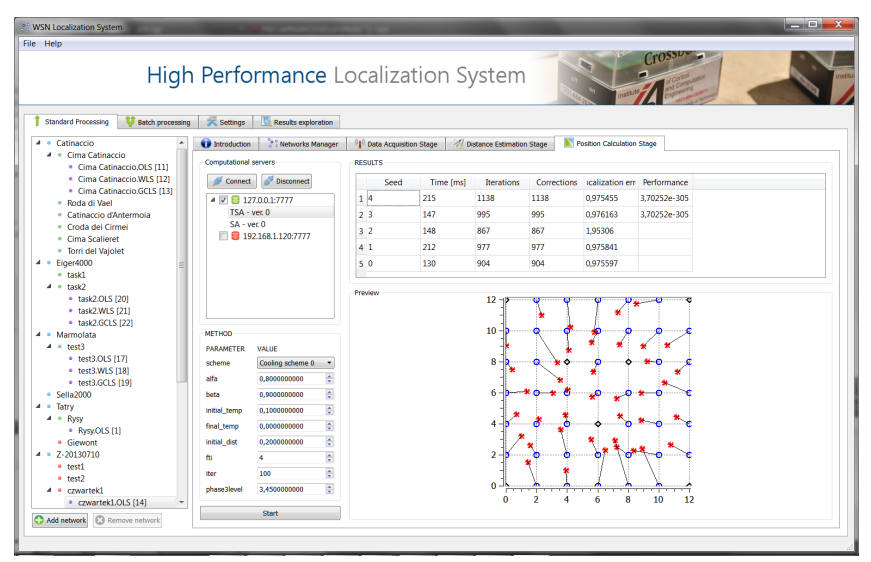

\subsection{Workflow in the HPLS}

Th HPLS provides framework whose main faces are: the real data acquisition from a workspace, the network model creation and calculation of positions of nodes in a network. During an experiment performed under the HPLS, one can distinguish two main stages: a preparatory stage and an experimental stage. The preparatory stage depends on the usage mode of the HPLS. In case of the on-line localization process all real data are gathered from the workspace and a network model is created and displayed. In case of the off-line testing and tuning of the localization schemes on synthetic networks the network topologies have to be generated. The user is asked to enter parameters concerned with the whole network (size of the deployment area, number of nodes, number of anchor nodes and their positions, radio communication range). Finally, the user is asked to configure the experiment - choose the localization algorithm and enter all parameters specific to this algorithm. The experimental stage begins when all decisions regarding the network system and the solvers are made and saved in the HPLS database. The Distance Estimation 
Figure 6 Workflow in the HPLS.

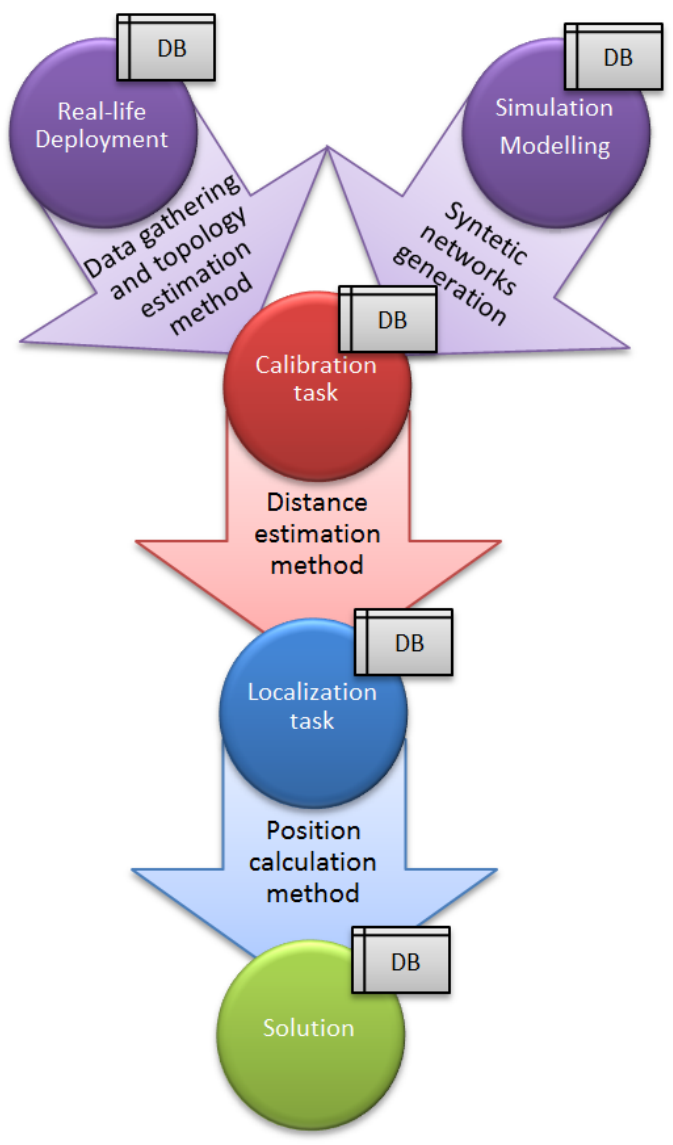

Module (DEM) is executed. The results of calculations, i.e., estimates of inter-node distances are stored in the database. Next, the Position Calculation Module (PCM) is executed. Final results of calculations, i.e., geographic coordinates of nodes with unknown location are displayed and stored. The workflow in the HPLS is depicted in Fig. 6.

\section{Numerical Verification and Performance Evaluation of HPLS}

All localization schemes implemented in the HPLS were validated through simulation. A number of tests were performed to cover a wide range of network system configurations including a size of a network (a number of nodes), a number of anchor nodes, a deployment of anchor nodes, a radio range and a RSSI measurement error. The metric for evaluating all considered localization schemes was the accuracy of the location estimates versus the deployment and computation cost. To compare the performance of the tested algorithms we used the mean error between the computed and the true location of each node in a workspace, defined as follows

$$
L E=\frac{1}{N} \cdot \frac{\sum_{i=1}^{N}\left(\left(x_{i}-\hat{x}_{i}\right)^{2}+\left(y_{i}-\hat{y}_{i}\right)^{2}\right)}{r^{2}} \cdot 100 \%
$$

Table 1 Basic characteristics of test networks.

\begin{tabular}{|l|c|c|c|c|c|}
\hline Network & Net1 & Net2 & Net3 & Net4 & Net5 \\
\hline$I$ & 200 & 500 & 1000 & 2000 & 4000 \\
\hline$N$ & 20 & 50 & 100 & 200 & 400 \\
\hline$C M$ & 15.00 & 23.40 & 13.35 & 16.64 & 14.17 \\
\hline \hline$n$ & 3.50 & 3.50 & 3.80 & 4.00 & 3.60 \\
\hline$\sigma$ & 3.20 & 3.20 & 3.20 & 3.50 & 4.00 \\
\hline$d_{0}$ & $1 \mathrm{~m}$ & $1 \mathrm{~m}$ & $1 \mathrm{~m}$ & $1 \mathrm{~m}$ & $1 \mathrm{~m}$ \\
\hline$P L\left(d_{0}\right)$ & $55 \mathrm{~dB}$ & $55 \mathrm{~dB}$ & $55 \mathrm{~dB}$ & $55 \mathrm{~dB}$ & $55 \mathrm{~dB}$ \\
\hline$n f[d B m]$ & -100 & -100 & -100 & -100 & -100 \\
\hline$P_{t}[d B m]$ & -3 & -7 & -3 & -10 & -7 \\
\hline area side & $100 \mathrm{~m}$ & $100 \mathrm{~m}$ & $200 \mathrm{~m}$ & $200 \mathrm{~m}$ & $500 \mathrm{~m}$ \\
\hline
\end{tabular}

where $\left(x_{i}, y_{i}\right)$ denote the true location of the sensor node $i,\left(\hat{x}_{i}, \hat{y}_{i}\right)$ is the estimated location of the sensor node $i$ and $r$ denotes the radio range. The location error $L E$ is expressed as a percentage error. It is normalized with respect to the radio range to allow comparison of results obtained for networks formed by various number of devices equipped with different transceivers.

The results of numerous experiments are presented and discussed in Niewiadomska-Szynkiewicz \& Marks (2009), Niewiadomska-Szynkiewicz (2012). In this paper we compare the performance of four localization schemes: trilateration (T), simulated annealing (SA) and two hybrid techniques TSA and TGA. The experiments were performed for synthetic WSNs (Net1 - Net5) consisting of 200 - 4000 nodes with randomly generated positions (both anchor and non-anchor nodes) in a square region. The networks were generated using NBM module tuned up using parameters obtained in the experimental field. Therefore $n$ and $\sigma$ parameters are on the level characterizing our experiments done outdoor, $d_{0}$ and $P L\left(d_{0}\right)$ as well as the noise level are constant. Since we considered much bigger networks then we are able to deploy in our testbed we used square side between 100 and 500 meters and transmission power level $\left(P_{t}\right)$ from values available for CC2420 radio chip (this radio chip offers 8 different levels of transmitters power equal: $0,-1,-3,-5,-7,-10,-15$ and $-25 \mathrm{dBm})$. Detailed characteristics of test networks, i.e. all mentioned parameters plus number of nodes $(I)$, number of anchor nodes $(M)$ and connectivity measure $(C M)$ are collected in Table 1 . The connectivity measure in Table 1 denotes the average number of connections between nodes in a given network.

For the purpose of simulations values of distances between neighboring nodes $i$ and $j$ in all synthetic networks were calculated according to the scheme described in section based on Low-power link modeling.

The averaged values of the localization errors $L E$ computed due to the formula (7) for Net1 - Net5, all tested localization systems and five executions of all tests are collected in Table 2. The results of calculations show that all localization systems based on stochastic optimization produce much more accurate localization with respect to the simple trilateration. A localization 
Table 2 Localization error $L E$ (various networks and algorithms)

\begin{tabular}{|l|c|c|c|c|c|}
\hline Network & Net1 & Net2 & Net3 & Net4 & Net5 \\
\hline T & 9.94 & 3.36 & 30.62 & 6.79 & 21.04 \\
\hline SA & 5.53 & 0.70 & 3.69 & 1.32 & 1.54 \\
\hline TSA & 0.78 & 0.41 & 0.76 & 0.44 & 0.83 \\
\hline TGA & 3.44 & 0.56 & 0.55 & 0.44 & 0.70 \\
\hline
\end{tabular}

Table 3 Calculation time in seconds (various networks and algorithms).

\begin{tabular}{|l|c|c|c|c|c|}
\hline Network & Net1 & Net2 & Net3 & Net4 & Net5 \\
\hline T & 0.01 & 0.03 & 0.14 & 0.52 & 2.36 \\
\hline SA & 2.71 & 5.41 & 24.69 & 63.77 & 211.93 \\
\hline TSA & 1.61 & 3.55 & 11.31 & 29.95 & 114.89 \\
\hline TGA & 5.75 & 15.08 & 28.88 & 78.80 & 273.55 \\
\hline
\end{tabular}

accuracy strongly depends on the applied optimization solver - in our experiments the SA algorithm gave better accuracy GA. The most promising approach seems to be the hybrid scheme TSA. Regardless of network size the localization error $L E$ has not exceeded $1 \%$. In some tasks TGA allows us to obtain better accuracy, however the results quality depends more on task structure.

The scalability of all considered localization systems is depicted in Table 3. The times used to localization of non-anchor nodes in five networks expressed in seconds are collected. As it was expected the calculation time for the simple trilateration scheme is almost unnoticeable as it does not exceed few seconds for every network from the testing set. The localization systems utilizing stochastic optimization require more extensive calculations and are thus much more time consuming. It is obvious that the computation time strongly depends on the size of given network and increases faster then linearly with the number of nodes. The TSA method is the fastest one but the differences between computation times of SA, TSA and TGA decrease for higher dimension networks.

\section{Testbed Validation}

The simulation is commonly used technique to verify the localization schemes and tune their parameters before the application in a real network. Unfortunately, the performance of the range based localization scheme strongly depends on the accuracy of inter-node distances estimation. Hence, it is very important to validate the developed system in testbed networks.

Therefore, a testbed networks were built and set up to facilitate tests of four localization schemes utilizing four methods: T, SA, TSA and TGA. Six testbed networks composed of 36 - 49 CM3000 motes (http://www.advanticsys.com/shop/mtmcm3000msp-p6.html) manufactured by Advantic Technology (clone of TelosB platform) and one base station were created. The architecture of the CM3000 mote is presented in Fig. 7. The operating system was TinyOS 2.1.2
Figure 7 The MTM-CM3000 mote architecture.

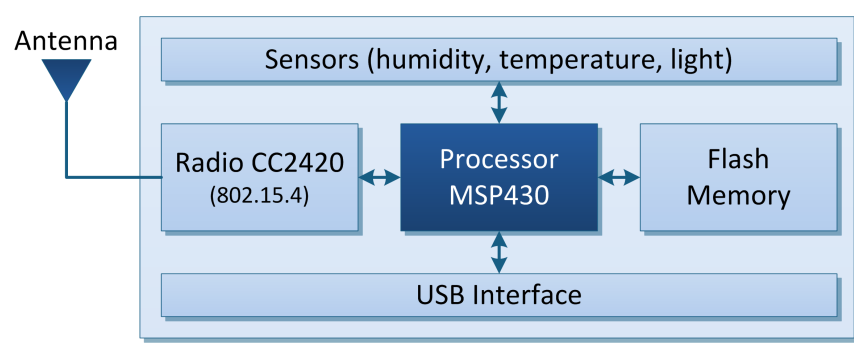

Figure 8 The Advantic CM3000 during deployment.

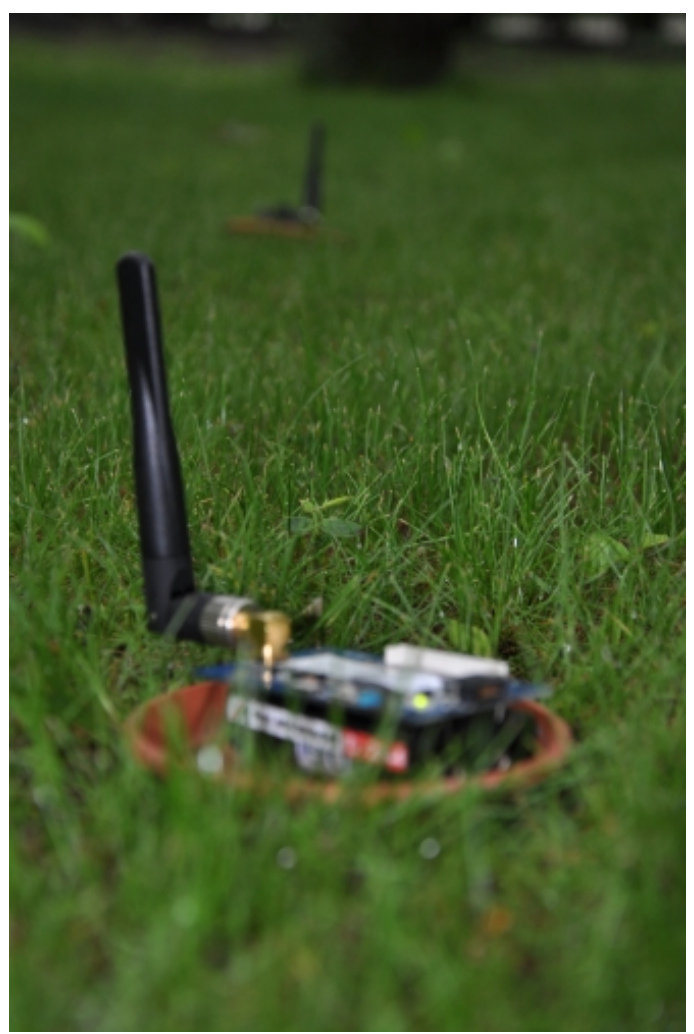

(www.tinyos.net/). It is an open source, highly portable operating system that can be used to on-line operation of WSN formed of real low-power wireless devices.

Photos from real-life deployments are presented in Figures 8 and 9. All of the experiments were done on a plain field $12 \mathrm{~m}$ by $12 \mathrm{~m}$. The transmission power was reduced to the lowest officially supported by Texas Instruments CC240 radio chip value equal $-25 \mathrm{dBm}$. Depending on experiment nodes were placed in a regular manner on a grid or in a random way.

As it was mentioned in section 4.3 the data collection procedure is based on Berkeley Low-power IP stack (IPv6). All the nodes forming experimental networks use the same image of operating system, which realizes tasks in the event-triggered manner. It means that there is no tasks to run periodically to send data to base station. The aim of such implementation is the energy conservation. As a consequence the base station needs to query all other motes about their image of network - how many neighbours do they have and how strength 
Figure 9 One of the tested topologies during outdoor testbed experiments.

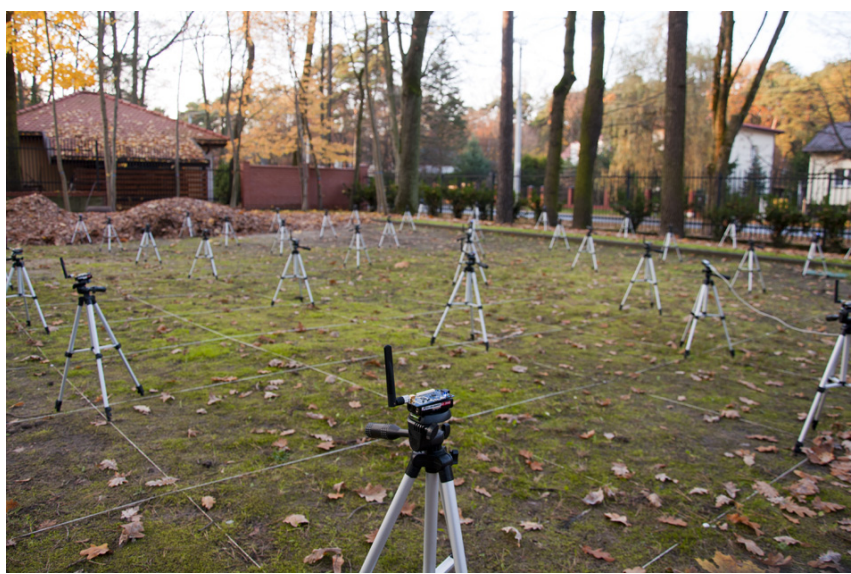

Table 4 Basic characteristics of testbed networks used for validation.

\begin{tabular}{|l|c|c|c|}
\hline Network & Net6 & Net7 & Net8 \\
\hline$I$ & 36 & 36 & 49 \\
\hline$M$ & 6 & 6 & 7 \\
\hline$C M$ & 28.22 & 28.27 & 21.69 \\
\hline
\end{tabular}

is the signal they are able to receive. This query can be propagated through the whole network using restricted flooding (flooding is restricted by setting appropriate value of TTL field) technique, so there is no need to send as many queries as there is motes in the network.

Each sensor node in a network which receives the request about its network image is responsible for exchanging packets with all nodes in its neighbourhood and collecting the vector of pairs of values neighbour $i d$ and RSSI measurement. RSSI readings are acquired using Texas Instruments CC2420 built-in Received Signal Strength Indicator providing a digital value that can be read from the 8 bit register. Next, these vectors are sent using multi-hop unicast transmission to the edge router and finally base station. Next these values are stored in the HPLS database.

Three testbed networks were selected in order to form a training set, which was used to tune up localization algorithms. Another three networks were used to validate the algorithms and quality of proposed solutions. The networks used for validation, with red stars marking the TSA solutions, are presented in Figure 10.

Basic characteristics of testbed networks, defined like in Table 1, are collected in Table 4.

The averaged values of the localization errors $L E$ computed due to the formula (7) for Net6 - Net8, TSA localization system and five executions of all tests are collected in Table 5. In the first row the localization error for data acquired from real-life deployments are presented. In the second row the localization errors for the same topologies, but radio signals modelled using NBM module are presented.
Figure 10 Real-life deployments used for localization algorithms validation.

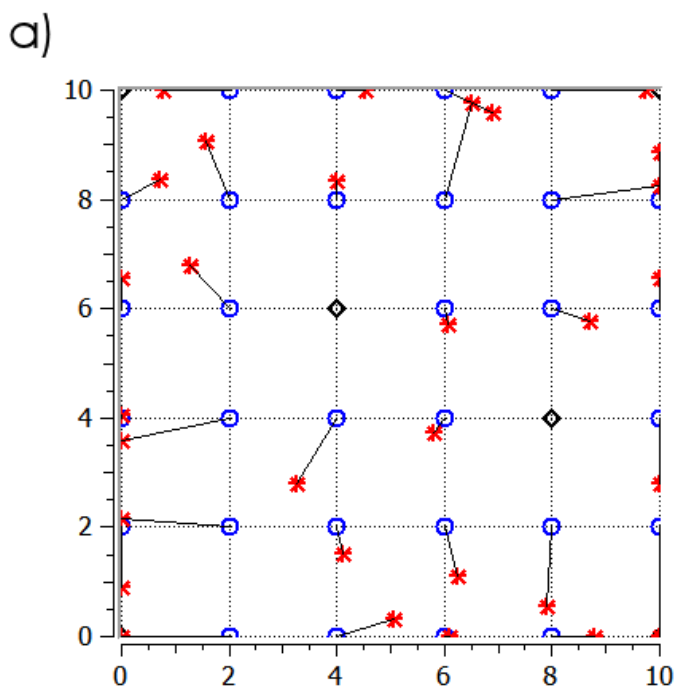

b)

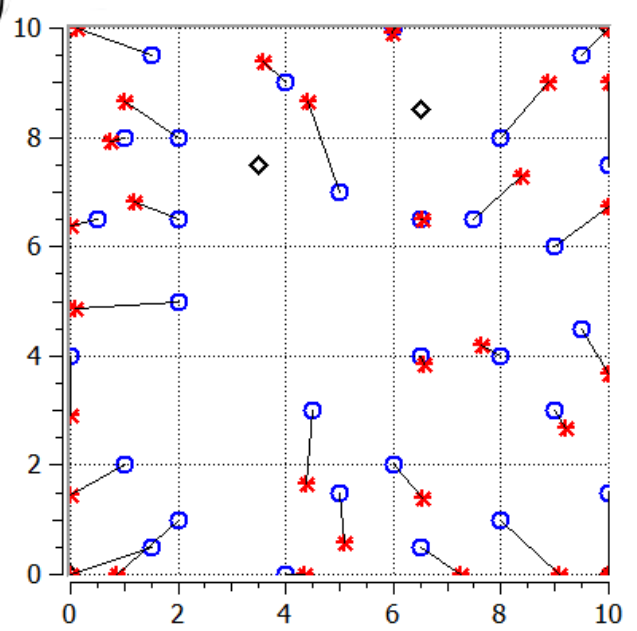

C)

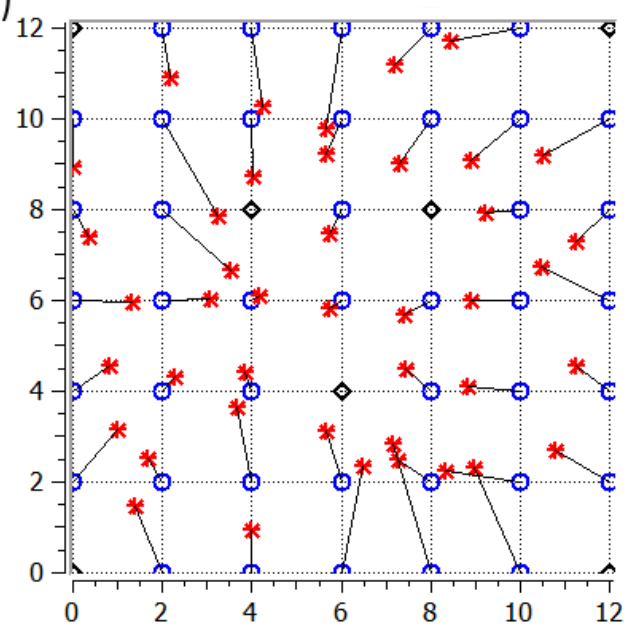

For all considered networks the LE don't exceed one percent, however the obtained results confirm that errors in RSSI readings obtained from real life deployments are greater than in case of radio signals modelling. 
Table 5 Localization error $L E$ (TSA for various testbed networks)

\begin{tabular}{|l|c|c|c|}
\hline Network & Net6 & Net7 & Net8 \\
\hline Testbed & 0.74 & 0.67 & 0.94 \\
\hline Simulation & 0.21 & 0.14 & 0.18 \\
\hline
\end{tabular}

\section{Summary and Concluding Remarks}

In the paper we describe and evaluate an integrated software framework High Performance Localization System, which supports sensor node localization starting from data gathering from real life deployments through signal propagation modeling to geographic coordinates calculation using various localization algorithms. We validated the localization schemes provided in the HPLS for various multihop network topologies through simulation and testbed implementation in our laboratory. The performance of all implemented localization algorithms was compared and discussed.

As a final observation, we can point that heuristic algorithms are efficient and versatile techniques that can be successfully used to localization of a WSN formed by physical devices. However, it is commonly known that to obtain a robust and efficient solver for WSN localization we have to tune all control parameters specific to this solver. It is time consuming process. The parallel implementation of the HPLS reduces the computing time and increases system scalability and robustness.

Although numerous localization systems are described in the literature, development of robust and scalable technique to estimate the location of sensor devices with high accuracy, minimal hardware cost and computational burdens is still a challenging task. The HPLS can support the design, development and evaluation of novel strategies and systems to localization in wireless sensor networks.

\section{Acknowledgement}

This work was partially supported by the National Science Centre grant NN514 672940.

\section{References}

Akyildiz, I. \& Vuran, M. (2010), Wireless Sensor Networks, John Wiley \& Sons, Ltd, West Sussex, UK.

Anderson, B., Mao, G. \& Fida, B. (2007), 'Wireless sensor network localization techniques', Computer Networks 51(10), 2529-2553.

Barsocchi, P., Lenzi, S., Chessa, S. \& Giunta, G. (2009), Virtual calibration for rssi-based indoor localization with ieee 802.15.4, in 'Communications, 2009. ICC '09. IEEE International Conference on', pp. $1-5$.

Benkic, K., Malajner, M., Planinsic, P. \& Cucej, Z. (2008), Using rssi value for distance estimation in wireless sensor networks based on zigbee, in 'Systems, Signals and Image
Processing, 2008. IWSSIP 2008. 15th International Conference on', pp. $303-306$.

Beutel, J. (2005), Handbook of Sensor Networks Compact Wireless and Wired Sensing Systems, CRC Press, Boca Raton.

Biswas, P. \& Ye, Y. (2004), Semidefinite programming for ad hoc wireless sensor network localization, in 'IPSN '04: Proc. of the third international symposium on Information processing in sensor networks', ACM Press, New York, NY, USA, pp. 46-54.

Chuang, P. \& Wu, C. (2008), An effective pso-based node localization scheme for wireless sensor networks, in 'Proc. of the 9th Inter. Conference on Parallel and Distributed Computing Applications and Technologies)', pp. 187-194.

Costa, J., Patwari, N. \& Hero, A. (2006), 'Distributed weighted-multidimensinal scaling for node localization in sensor networks', ACM Transaction Sensor Networks 2(1), 39-64.

Farooqi, A. H. \& Khan, F. A. (2012), 'A survey of intrusion detection systems for wireless sensor networks', Int. J. of Ad Hoc and Ubiquitous Computing 9(2), 69-83.

Goldberg, D. E. (1989), Genetic Algorithms in Search, Optimization and Machine Learning, Studies in Applied Mathematics, Addison-Wesley.

Ji, Y. (2011), 'Performance analysis for indoor location determination', Int. J. of Ad Hoc and Ubiquitous Computing 8(1), 3-15.

Kannan, A. A., Mao, G. \& Vucetic, B. (2005), Simulated annealing based localization in wireless sensor network, in 'LCN '05: Proceedings of the The IEEE Conference on Local Computer Networks 30th Anniversary', IEEE Computer Society, Washington, USA, pp. 513-514.

Kannan, A., Mao, G. \& Vucetic, B. (2006), Simulated annealing based wireless sensor network localization with flip ambiguity mitigation, in '63rd IEEE Vehicular Technology Conference', pp. 1022-1026.

Karl, H. \& Willig, A. (2005), Protocols and Architectures for Wireless Sensor Networks, Wiley.

Lee, J., Chung, W. \& Kim, E. (2010), 'A new rangefree localization method using quadratic programming', Computer Communications 34, 998-1010.

Li, C., Li, Y., Shen, Y., Liu, L. \& Cao, Q. (2010), An optimization algorithm for wireless sensor networks localization using multiplier method, in 'Proc. of the Third Inter. Joint Conference on Computational Science and Optimization', IEEE Computer Society, China, pp. 337-341.

Mao, G. \& Fidan, B. (2009), Localization Algorithms and Strategies for Wireless Sensor Networks, Information Science Reference, Hershey, USA.

Mao, G., Fidan, B. \& Anderson, B. D. O. (2007), 'Wireless sensor network localization techniques', Computer Networks: The International Journal of Computer and Telecommunications Networking 51(10), 2529-2553.

Marks, M. \& Niewiadomska-Szynkiewicz, E. (2011), Self-adaptive localization using signal strength measurements, in 'SENSORCOMM 2011, the Fifth International Conference on Sensor Technologies and Applications', IARIA, Nice, France, pp. 73-78. 
Medidi, M., Slaaen, R., Zhou, Y., Mallery, C. \& Medidi, S. (2006), Cluster-based localization in wireless sensor networks, in 'Proc. of SPIE, Wireless Sensing and Processing'.

Motter, P., Allgayer, R., Mller, I. \& de Freitas, E. (2011), Practical issues in wireless sensor network localization systems using received signal strength indication, in 'Proc. of Sensors Applications Symposium (SAS)', IEEE Computer Society, San Antonio, USA, pp. 227-232.

Niculescu, D. \& Nath, B. (2001), Ad hoc positioning system (aps), in 'GLOBECOM: Global Telecommunications Conference', Vol. 5, San Antonio, USA, pp. 2926-2931.

Niewiadomska-Szynkiewicz, E. (2012), 'Localization in wireless sensor networks: Classification and evaluation of techniques', International Journal of Applied Mathematics and Computer Science 22(2), 281-297.

Niewiadomska-Szynkiewicz, E. \& Marks, M. (2009), 'Optimization schemes for wireless sensor network localization', International Journal of Applied Mathematics and Computer Science 19(2), 291-302.

Ramadurai, V. \& Sichitiu, M. L. (2003), Localization in wireless sensor networks: A probabilistic approach, in 'Proceedings of International Conference on Wireless Networks (ICWN 2003)', Las Vegas, USA, pp. 300-305.

Rappapport, T. (2002), Wireless communications: principles and practice, Communications Engineering and Emerging Technologies Series, second edition edn, Prentice Hall.

Rodrigues, J. J. \& Neves, P. A. (2010), 'A survey on ip-based wireless sensor network solutions', International Journal of Communication Systems 23(8), 963-981.

Sarigiannidis, G. (2007), Localization For Ad Hoc Wireless Sensor Networks, Paperback, LL.

Savvides, A., Han, C. \& Strivstava, M. (2001), Dynamic finegrained localization in ad-hoc networks of sensors, in 'Proc. of ACM MobiCom', Rome, Italy, pp. 166-179.

Sayadnavard, M., Haghighat, A. \& Abdechiri, M. (2010), Wireless sensor network localization using imperialist competitive algorithm, in 'Proc. of the 3rd IEEE Inter. Conference on Computer Science and Information Technology)'.

Shang, Y., Ruml, W., Zhang, Y. \& Fromherz, M. (2004), 'Distributed weighted-multidimensinal scaling for node localization in sensor networks', IEEE Transactions on Parallel and Distributed Systems 15(11), 961-674.

Shekofteh, S., Yaghmaee, M., Khalkhali, M. \& Deldari, H. (2010), Localization in wireless sensor networks using tabu search and simulated annealing, in 'Proc. of the 2nd Inter. Conf. on Computer and Automation Engineering (ICCAE)', IEEE Computer Society, Singapore, pp. 752757.

Shu, J., Zhang, R., Liu, L., Wu, Z. \& Zhou, Z. (2009), 'Cluster-based three-dimensional localization algorithm for large scale wireless sensor networks', Journal of Computers 4(7), 585-592.

Silva, R., Silva, J. S. \& Boavida, F. (2009), 'Evaluating 6lowpan implementations in wsns', Proceedings of 9th Conferncia sobre Redes de Computadores Oeiras, Portugal pp. 1-5.

Srinivasan, K. \& Levis, P. (2006), Rssi is under appreciated, in 'In Proceedings of the Third Workshop on Embedded Networked Sensors (EmNets)', Cambridge, USA.
Tam, V., Cheng, K. \& Lui, K. (2006), A descentbased evolutionar approach to enhance position in wireless sensor networks, in 'Proc. of the 18th IEEE Inter. Conference on Tools with Artificial Intelligence (ICTAI'06)', pp. 568-574.

Tseng, P. (2007), 'Second-order cone programming relaxation of sensor network localization', SIAM Journal on Optimization 18, 156-185.

Vecchio, M., Lopez-Valcarce, R. \& Marcelloni, F. (2012), 'A two-objective evolutionary approach based on topological constraints for node localization in wireless sensor networks', Applied Soft Computing 12, 18911901.

Vo, N., Vo, D., Challa, S. \& Lee, S. (2008), Nonmetric mds for sensor localization, in 'Proc. of Inter. Symposium on Wireless Pervasive Computing', pp. 396-400.

Wang, Z., Bulut, E. \& Szymanski, K. (2010), 'Distributed energy ecient target tracking with binary sensor networks', ACM Transaction on Sensor Networks 6(4), 1 -32 .

Wang, Z., Zheng, S., Ye, Y. \& Boyd, S. (2008), 'Further relaxation of the semidefinite programming approach to sensor network localization', SIAM J. Optim. 19, 655673.

Wessels, A., Wangb, X., Laurb, R. \& Langa, W. (2010), 'Dynamic indoor localization using multilateration with rssi in wireless sensor networks for transport logistics', Procedia Engineering 5, 220-223.

Zuniga, M. \& Krishnamachari, B. (2004), Analyzing the transitional region in low power wireless links, in 'In First IEEE International Conference on Sensor and Ad hoc Communications and Networks (SECON)', Santa Clara, USA, pp. 517-526. 